However, the effects and mechanism of $L G G$ on bowel function remains unclear. 5-Hydroxytryptamine4 Receptor is a critical receptor relating to the intestine motility and secretion function. In this study, we aimed to investigate whether $L G G$ could improve the defecation function via upregulating 5HT4R and modulating gut microbiota in mice.

Methods Male C57BL/6 mice 6-8 weeks in age were randomly divided into $\underline{3}$ groups: MRS group $(n=10)$, Tegaserod group (positive control, $\mathrm{n}=15)$ and $L G G$ group $(\mathrm{n}=15)$, and MRS broth, tegasromide maleate and $L G G$ supernatant were gavaged respectively for $\underline{Z}$ days. YAMC cells and Caco 2 cells were used for experiment in vitro. Defecation parameter including the number of pellets in 2 hours, fecal weight, fecal dry weight, fecal water content, and the gastrointestinal transit time (GITT) were detected. PAS and AB-PAS staining were used to evaluate goblet cells number in mice colon, and 5HT4R and MUC2 expression were determined Real-time PCR and Western blotting in vivo and in vitro. Gut microbiota and short-chain fatty acid were analyzed by 16 sRNA pyrosequencing analysis and gas chromatography method.

Results The number of defecation pellets in $2 \mathrm{~h}$, fecal weight, fecal dry weight and fecal water content in the Tegasromide group and $L G G$ group were significantly increased compared with those in the MRS group, PAS staining showed that the average number of goblet cells in Tegasromide group and $L G G$ group were significantly increased in mice colon sections compared with MRS group. AB-PAS showed increased cavitated goblet cells in the $L G G$ group, and the mRNA and protein levels of 5-HT4R and MUC2 were upregulated both In vitro and in vivo. In this study, increased levels of Alistipes, Allobaculum, and Desulfovibrioin were found in the LGG group which have been reported to be involved in intestine motility, intestinal barrier.

Conclusions $L G G$ supernatant could improve defecation function in mice accompanied by upregulating 5-HT4R and MUC2 production, and modulating gut microbiota. Thus, this study will provide a better understanding of probiotics for the prevention and treatment of constipation.

\section{IDDF2019-ABS-0301 MAPPING OF GUT MICROBIOME SECRETOME IN COLORECTAL CANCER: A MALAYSIAN DATA}

${ }^{1}$ Siok-Fong Chin*, 'Putri Intan Hafizah Megat Mohd Azlan, '2Luqman Mazlan, ${ }^{1}$ Huimin Neoh, ${ }^{3}$ Raja Affendi Raja Ali, ${ }^{1}$ Rahman Jamal. 'UKM Medical Molecular Biology Institute, Universiti Kebangsaan Malaysia, Kuala Lumpur, Malaysia; ${ }^{2}$ Department of Surgery, Faculty of Medicine, Universiti Kebangsaan Malaysia, Kuala Lumpur, Malaysia; ${ }^{3}$ Department of Medicine, Faculty of Medicine, Universiti Kebangsaan Malaysia, Kuala Lumpur, Malaysia

\subsection{6/gutjin-2019-IDDFabstracts.67}

Background The human gut is home to trillions of gut flora that thrive in a delicate balance, which has helped maintain the host's gut homeostasis and mutually benefited both parties tremendously. However, a drastic perturbation of microbial composition has hampered gut homeostasis initiating tumour microenvironment for the development of colorectal cancer (CRC). The objective of this study was to profile secreted proteins released from the human gut and microbial of CRC patients and control with healthy colon morphology by assessing the secretome in stool samples, using mass spectrometry technology.

Methods Stool samples from 26 CRC and 20 controls were collected, homogenized and filtered prior to protein extraction and analysis. Samples were subjected to in-solution digestion, followed by protein identification and quantification. Bioinformatics tools such as SPSS, MaxQuant, DAVID and String were used for statistical analysis, data visualization, functional annotations and prediction of protein interactions and pathways.

Results We identified more human origin proteins in CRC as compared to control \& inversely for proteins from microbial origin. The identified human exclusive proteins for CRC were mostly related to protein binding function and the top expressed proteins were mapped to Stage I and II CRC. The best prediction model was built upon the combination of human Huntingtin \& RNA exonuclease 5 proteins. The model was sensitive but not specific in discriminating control from CRC. Meanwhile, the top annotated KEGG pathway for human CRC-exclusive proteins was Hypoxia-inducible factor-1 (HIF-1). In addition, yeast proteins were topping the microbial CRC-exclusive proteins list, with the predicted protein interactions mapped to DNA repair, transcription regulation \& ATP binding.

Conclusions In conclusion, gut flora and human colon released an abundance of microbial proteins to the external environment possibly mediating various host-microbial reactions and responses in CRC.

\section{IDDF2019-ABS-0303 METHYLATION PROFILES OF PHANTOM $\underline{5}$ ENHANCER AND OPEN CHROMATIN IN COLORECTAL CANCERS}

${ }^{1}$ Muhiddin Ishak*, ${ }^{1}$ Najwa Farhah Mohd Yusof, ${ }^{1}$ Sazuita Saidin, ${ }^{2}$ ssa Mohamed Rose, ${ }^{3}$ Luqman Mazlan, ${ }^{3}$ Ismail Sagap, ${ }^{1}$ Rahman Jamal, ${ }^{1}$ Nadiah Abu, ${ }^{1}$ Nurul Syakima Ab Mutalib. 'UKM Medical Molecular Biology Institute (UMBI), National University of Malaysia, Kuala Lumpur, Malaysia; ${ }^{2}$ Department of Pathology, Faculty of Medicine, National University of Malaysia, Kuala Lumpur, Malaysia; ${ }^{3}$ Department of Surgery, Faculty of Medicine, National University of Malaysia, Kuala Lumpur, Malaysia

\subsection{6/gutjnl-2019-IDDFabstracts.68}

Background Colorectal cancer (CRC) contributes around 1.36 million of the total cases worldwide and it has become evident over the past two decades that epigenetic alterations also play key roles in CRC pathogenesis. Majority of the research epigenetic alterations has examined the promoter regions, while other loci such including enhancers and open chromatins are not yet well described. Hence, this study aims to specifically profile the methylome of enhancers and open chromatins in CRC

Methods Genomic DNA and total RNA were extracted from cancer-adjacent normal colonic tissues and subjected to bisulfite conversion and cDNA synthesis, respectively. DNA methylation analysis was performed using Human Infinium Epic Beadchip Array which includes $>23,000$ enhancers and $>461,000$ open chromatins. Microarray data were analyzed using Genome Studio V1.8 and Bioconductor-ChAMP V2.8.1. The differentially methylated regions were validated via bisulfite conversion, cloning, and sequencing of individual clones. In order to correlate the effect of DNA methylation at the specific loci, the gene expression of the differentially methylated loci was analysed using quantitative real-time PCR

Results We identified 342 significant differently methylated enhancers and 2187 significant differentially methylated open chromatin. There were 192 hypermethylated and 150 hypomethylated enhancers compared to 1110 hypermethylated and 1076 hypomethylated open chromatins. Pathway enrichment 
analysis reveals that the majority of differentially methylated genes in the enhancer region are involved in the cancer and focal adhesion pathway. In addition, the pathways in cancer and PI3K-Akt signaling are significantly enriched in the differentially methylated open chromatin loci. Significant differentially methylated enhancer and open chromatin loci, OPLAH cg26256223 (hypermethylated open chromatin) and LYN cg08621168 (hypomethylated enhancer) were selected for further validation. qPCR analysis further confirmed the decrease of OPLAH gene expression level and vice versa for the LYN gene

Conclusions This is the first insight on the enhancers and open chromatins methylation profile in Malaysian CRC patients. The new knowledge from this study can be utilized to further increase our understanding of CRC methylomics, particularly on the enhancers and open chromatins. The functional roles of OPLAH cg26256223 and LYN cg08621168 warrant future investigations

\section{IDDF2019-ABS-0307 LONG NON-CODING RNA TMPO-AS1 REGULATES OESOPHAGEAL SQUAMOUS CELL CARCINOMA METASTASES THROUGH ACTIVATING GLI1 BY MAINTAINING LAP2A EXPRESSION}

Xiao-Jing Luo*, Jia Liu, Huai-Qiang Ju, Rui-Hua Xu. State Key Laboratory of Oncology in South China, Collaborative Innovation Center for Cancer Medicine, Sun Yat-sen University Cancer Center, China

\subsection{6/gutjnl-2019-IDDFabstracts.69}

Background Long non-coding RNAs (lncRNA) have been found to play important regulatory roles in cancer development and progression. However, functional lncRNAs and their downstream mechanisms remain largely unknown of oesophageal squamous cell carcinoma (OSCC) metastases. We aimed to identify lncRNAs that regulate OSCC metastases and investigate their downstream mechanisms.

Methods Small interfering RNA library was built of the top 50 overexpressed lncRNAs in OSSC according to TCGA database. Transwell migration assay was performed to identify the lncRNA that markedly affected cell migration. TMPO-AS expression was validated by $\mathrm{qPCR}$ in patient tissues and OSSC cell lines. Gain and loss of function of TMPO-AS 1 were performed in transwell migration and invasion assays in vitro. Lung and lymph node metastases models were built with short hairpin RNA knockdown in vivo. RNA pull-down and RNAase protection assay (RPA) followed by $\mathrm{qPCR}$ analysis identified the RNA-RNA interaction. Downstream mechanisms were examined by regular molecular biological methods.

Results We identified TMPO-AS 1 as a key regulator of OSSC metastases. TMPO-AS 1 expression was upregulated in OSSC tumor tissues compared to adjacent normal tissues and positively correlated with the mRNA expression of TMPO (LAP2), its sense coding gene. Knockdown of TMPO-AS 1 significantly inhibited OSSC cells migration and invasion in vitro and attenuated metastases in two different animal models in vivo. Overexpression of TMPO- $A S_{1}$ showed contrary effects. RNA pull-down identified the interaction between TMPO-AS mRNA and LAP2a mRNA. RPA assay further confirmed $T M P O-A S_{1}$ 's protective effect on $L A P 2 a$ mRNA. Western blotting found that knockdown of TMPO-AS ${ }_{1}$ decreased the expression of LAP2a without affecting LAP2b. Ectopic expression of LAP2a after TMPO-AS $S_{1}$ knockdown rescued the adverse effect on cell migration and invasion. By regulating the expression of its sense coding gene LAP2, TMPO-AS maintained the levels of LAP2a, which in turn activated Hedgehog signaling transcription factor $\mathrm{GLI}_{1}$ and its downstream target SNAIL, therefore promoting OSSC progression. Conclusions TMPO-AS $S_{1}$ acts as an essential regulator in OSSC metastases by interacting with $L A P 2 a$ mRNA and maintaining its levels, which activates the LAP $2 a-G_{1} I_{1}-S N A I L$ axis and facilitates OSSC metastases.

\section{IDDF2019-ABS-0312 THE LANDSCAPE OF RECURRENT NONCODING MUTATIONS IN COLORECTAL CANCERS}

${ }^{1}$ Nurul-Syakima Ab Mutalib*, ${ }^{1}$ Ryia Illani Mohd Yunos, ${ }^{1}$ Najwa Farhah Mohd Yusof, ${ }^{1}$ Muhiddin Ishak, ${ }^{1}$ Sazuita Saidin, ${ }^{2}$ Isa Mohamed Rose, ${ }^{3}$ Luqman Mazlan, ${ }^{3}$ Ismail Sagap, ${ }^{1}$ Rahman Jamal. 'UKM Medical Molecular Biology Institute (UMBI), National University of Malaysia, Malaysia; ${ }^{2}$ Department of Pathology, Faculty of Medicine, National University of Malaysia, Malaysia; ${ }^{3}$ Department of Surgery, Faculty of Medicine, National University of Malaysia, Malaysia

\subsection{6/gutjnl-2019-IDDFabstracts.70}

Background Colorectal cancer (CRC) is among major cancer worldwide, and it has become evident that the identification of driver mutations is fundamental to understanding carcinogenesis. Although genes frequently mutated in CRC have been identified, those pursuits for driver mutations have mainly focused on the coding genome. The noncoding somatic mutation landscape remains unexplored. Hence, this study aims to characterize the landscape of noncoding somatic mutations in CRC.

Methods Genomic DNA was extracted from 36 cancerous colonic tissues and subjected to whole genome sequencing (WGS). Blood DNA served as the germline control. The sequencing data were aligned to hg19 using Burrow-Wheeler Aligner (BWA), somatic variants were called using muTect2 and the noncoding mutations were prioritized using funseq2. Pathway enrichment analysis was performed using DAVID, and gene expression data were retrieved from Firebrowse.

Results We identified 72,890 recurrent noncoding alterations, which were altered in at least 2 patients. Focusing on the distal regulatory modules (DRMs), the majority (83.2\%) of the alterations were identified in transcription factor binding peaks (TFP), followed by Segway/ChromHMM-predicted enhancers (8.39\%) and DNase I hypersensitive sites (DHS) (3.68\%). In addition, $0.56 \%$ alterations were discovered in the long intergenic noncoding RNAs (lincRNAs) and $0.02 \%$ in transcription factor bound motifs in peak regions (TFM). MAFK| chr4:50489-63382 and WRNIP1|chr6:292554-300803 are the most frequently altered TFPs (9/36), while drm chr17:21514800-21518300 is the most frequently altered enhancers (8/36). There was a modest upregulation of MAFK $(\log 2$ RSEM $=0.997)$ and WRNIP1 $(\log 2$ RSEM $=1.45)$ gene expression in cancer compared to the normal based on 626 CRCs from COADREAD TCGA dataset. LincRNA (ENSG00000238261.3[BX004987.5]) alteration was identified in $8 / 36$ of patients. We also show the commonality of pathways targeted by coding and noncoding mutations, demonstrated by TP53, APC, and KRAS, which regulates Wnt and MAPK signaling, the crucial pathways in colorectal carcinogenesis.

Conclusions This study provides an enhanced understanding of colorectal carcinogenesis and describes the advantages of 